\title{
How Our Body's Evolution was guided by Our Abdominal Organs (The Intelligence behind Evolution)
}

\author{
Fletcher Kovich, Lic.Ac* (D) \\ Curepoint Acupuncture, Bristol, UK
}

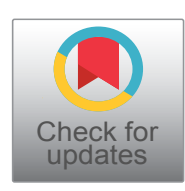

\begin{abstract}
A current theory is that each creature's evolution may have been determined by random variations in its genes, combined with natural selection, and perhaps some other unknown influence.

In contrast, this article argues that a creature's senses, the shape of its body, and whatever thoughts or emotions it now has may have all evolved in response to information from its own abdominal organs.
\end{abstract}

\section{Keywords}

Acupuncture, Chinese medicine, COVID19, Evolution, Meridian

\section{Introduction}

Within all living creatures, there exists an electrical current, probably conducted via semi conduction through its connective tissue network [1]. Upon this current is superimposed information from the functioning of the creature's abdominal organs [2].

This happens by accident, rather than being by design, just as air molecules accidentally transfer information in the form of sound. The information conveyed in sound can be easily transferred to an electrical current, using a microphone. The electrical current then contains complex information from the atmosphere surrounding the microphone. In a similar fashion, biological information from the function of each organ is superimposed on the body's electrical current. The information is live, changes at least every millisecond, and indicates such things as the overall strength of the organ function, its muscular activity, and possibly many other states $[2,3]$.

It has been demonstrated that this organ information conveyed on electromagnetic waves, is interpreted by some aspect of all bodily tissue, causing that tissue to make changes in itself to reflect that information [2,3]. This is called the intelligent tissue theory, and it explains how such things as acupuncture work.

When an organ is stressed (that is, malfunctioning in some way), the organ's state is communicated on electromagnetic waves to every cell in the body. Tracts of tissue in certain locations have evolved to mainly interpret information from one particular organ, and locations along that tract of tissue become stressed when the associated organ is stressed. These tracts of tissue are known as the meridians [1].

Hence, when the lungs are stressed in some way, certain points along the lung meridian will also become immediately stressed. If these points are pressed, they will feel tender. The local tissue feels as though it had been bruised, simply because something in its structure is adopting the stress that currently exists in the lungs.

The magical thing about this accidental mechanism is that it also works in reverse. If one of these local points is stimulated, such that the local tissue returns to normal function, because of the association of this point with the related organ, that organ is persuaded to also return to normal function [1]. This is how acupuncture works to immediately correct organ malfunction; and some types of massage can work in the same way. This explains why people feel so deeply relaxed after acupuncture or massage. They feel that way because their main organs have released their stress.

This mechanism usually corrects organ malfunctions within around one second, which can be demonstrated as follows. These key locations (acupoints) are usually used as diagnostic points. If the point feels tender when pressed, this means the related organ is stressed. And when the organ is treated with acupuncture, using a different key acupoint, and that initial "diagnostic" acupoint is pressed again, the patient usually

*Corresponding author: Fletcher Kovich, Lic.Ac, Curepoint Acupuncture, Bristol, UK

Accepted: October 17, 2020

Published online: October 19, 2020

Citation: Kovich F (2020) How Our Body's Evolution was guided by Our Abdominal Organs (The Intelligence behind Evolution). Insights Anthropol 4(2):290-293 
reports that it is no longer tender. This transformation happens because the local tissue at that location is interpreting the information from the related organ, and the organ has returned to normal function (is no longer stressed). Even though this "diagnostic" point was not needled, this transformation in itself often occurs within about one second of the other related acupoint being needled. This suggests that the organ malfunction was corrected within around one second; which is usually confirmable by other diagnostic signs: in the organ "pulses" (which are subjective) and in colour changes at locations on the patient's tongue that relate to that organ (which are objective). And this process also demonstrates the responsiveness of the local tissue at these meridian locations to any real-time changes in organ function.

\section{But How Did the Tissue along These Tracts, Evolve to Interpret Only Information from One Particular Organ?}

We know that bodily tissue now interprets organ information carried on electromagnetic waves [2,3], but did this ability always exist, throughout evolution?

There are two possible scenarios. The first is that the body became fully evolved, and then the tissue along each meridian location then spontaneously developed the ability to only interpret information from one particular organ. This seems unlikely, and there would be no reason for this arrangement to occur. The second, much more likely scenario, is that, from the earliest stages of evolution, bodily tissue already had this ability to interpret organ information conveyed on electromagnetic waves. And as the digestive organs first began to evolve, the body around those organs interpreted this new organ information, and over millions of years this caused the body to gradually adopted the particular shape it did-in response to that information.

There is clear evidence that supports this scenario. If our twelve abdominal organs are considered in terms of their relationships to each other (their abdominal location, mass, the substances they process, and how directly those substances communicate with the outside), they can all be placed in a definite order, producing a table of hierarchy. This is shown in Table 1.

The scale used in Table 1 to order the organs is that of "Yin to Yang," which can be viewed as a simple statement of polarity (negative to positive) but considered in relation to many factors, such as an organ's mass, its geographic location in the body, how directly or indirectly an organ communicates with the outside (for example, the lungs process air, which communicates directly with the outside; this makes the lungs more Yang than the heart-because the heart processes blood, which does not communicate directly with the outside), and similar qualities [1].

Figure 1 shows the position of the meridians on the left arm, inner and outer surfaces. If the geographical location of each meridian is compared with the organ hierarchy shown in Table 1 , it is found that the position of every meridian exactly matches the ranking of its related organ in the hierarchy of organs shown in Table 1.

For example, if we consider the topography of the arm's inner surface, and apply the "Yin-Yang" scale to that surface, the progression is from Yin to Yang running across the arm, from the back edge to the front edge (Figure 1). Positioned across this surface are the meridians for the heart, pericardium and the lungs, in that order. The heart meridian is placed on the most Yin part of this surface; the pericardium meridian is placed on a more Yang part than the heart meridian is placed on; and the lung meridian is placed on the most Yang part of this surface.

The relative positioning of these three meridians corresponds exactly with the organ hierarchy shown in Table 1. It is as though that hierarchy has been used to determine where the meridians would be located on the body (or, in other words, to determine which geographical tracts on the body would reflect the functional states in each organ). And this pattern is true for all meridians, including whether they appear on the inner or outer surface of a limb, and whether they are placed on the arms or the legs. Further, the meridians also

Table 1: The "Yin-Yang" hierarchy of the organs.

Left column = Yin (inner); right column = Yang (outer)

\begin{tabular}{|l|l|l|}
\hline Lungs & Large intestine & \\
\hline Pericardium & Lymphatic system & Meridians on the arms \\
\hline Heart & Small intestine & \\
\hline (Pancreas) & Stomach & Meridians on the legs \\
\hline (Liver) $^{1}$ & Gallbladder & \\
\hline "Kidneys"2 & Bladder & \\
\hline
\end{tabular}

Caption: There are two columns and six rows. Organs in the left column are Yin when compared to organs in the right column. And if an organ appears in a row that is lower than the row another organ appears in, the organ in the lower row is more Yin than the organ in a higher row, and vice versa: the higher organ is more Yang than the lower organ.

${ }^{1}$ The pancreas and liver are bracketed, because their position in the table is interchangeable, depending on the bodily location being considered. It is for this reason that these two meridians cross one another at three different locations on the body, indicating that the "YinYang" polarity of these two organs is crossing over at those three locations.

${ }^{2}$ In Chinese medicine, whenever the "kidneys" are mentioned, this also includes the adrenal glands and sex organs, all considered as a single overall organ. 


\section{Yang (front edge)}

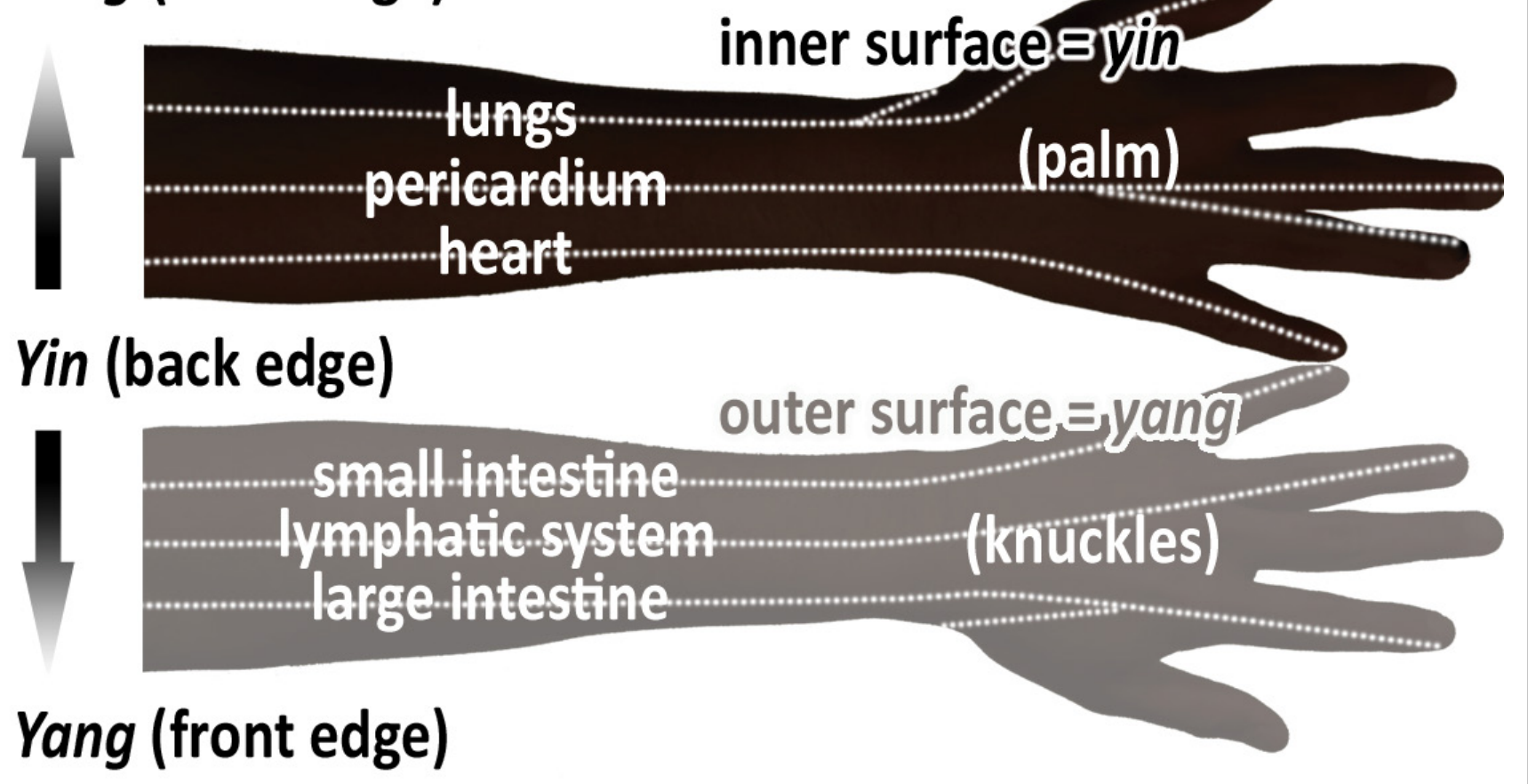

Figure 1: The inner and outer surfaces of the left arm, showing the meridian locations along the arm.

exist on the torso; and each meridian's exact route across the torso, also reflects the function of its corresponding organ [1].

The meridians do not have a structure of their own. They are simply tracts of normal bodily tissue that reflect states in their related organ [1]. It is as though this normal bodily tissue, initially reflected the functions of all the organs, and over millions of years, because there was a clear hierarchy between the organs, this caused the bodily tissue to gradually organize itself into the shape of our body today, such that all the geographical surfaces were then arranged to reflect this organ hierarchy. In other words, because there was clear information in these body-wide electromagnetic waves, and all the bodily tissue could interpret this information, over time, the bodily tissue made sense of this information by reshaping and reorganizing itself.

And because a particular tract of tissue evolved into the geographical location that it did by interpreting the information from one particular organ, that tract of tissue now only responds to information from that same organ.

This influence is so responsive that even within a person's lifetime, when one of their main organs is consistently stressed, this can produce a deformity of their torso that reflects this, such as a bump on their torso in the location of that organ's meridian or one of its main acupoints. This process is like a snapshot of the body evolving in response to the organ functions, but doing this within a person's lifetime.

Our Organs May Have Also Influenced the Evolution of Our Senses

Each of our senses is associated with a particular or- gan, and the good function of that sense relies on the good function of its related organ. For example, when a person's "kidney" function is poor, they cannot hear well; when their pancreas function is poor, they cannot taste; when their lung function is poor, they cannot smell; when their liver is stressed, they cannot see clearly; and when their heart function is poor, their speech is affected [1].

When considering the nature of each of these organ functions, it is possible to identify the motivation within that organ (in other words, the organ's "intention"). And it is possible to understand how this same motivation may have caused the sense related to that organ to evolve. For example, the lungs' motive is to take in air from outside, separate the oxygen, incorporate this and expel the waste products of respiration. The sense associated with the lungs is the sense of smell. Having this sense, supports the lungs' motive, since it is then able to smell the quality of the air it is breathing, and avoid breathing in bad air. When the lung function is poor, this greatly diminishes a person's sense of smell. The sense's dependence on the lungs' physiology suggests that this physiology may have been present from the start of the sense's evolution; and hence supports the notion that the sense may have evolved in response to this physiology. In other words, that it was the lungs' motivation (its desire to perform its main task) that caused the sense of smell to evolve, which would then help the lungs better perform its purpose.

This same argument can be applied to all five main organs, and suggests that each sense may have evolved under the influence of its related abdominal organ. This would explain why the good function of each sense organ now depends on the good function of its related abdominal organ. 


\section{Even Our Thoughts are Influenced by Our Organs}

There is a similarity between the physical function of each organ and certain thought patterns [1]. And when one of those thought patterns is blocked in our mind, this causes the physical function of the corresponding organ to also be blocked, which then produces a whole range of physical, mental and emotional symptoms in us [1].

The author speculates that because our organs had existed for millions of years before our conscious mind started having complex thoughts, that the already-existing logic of those organ functions (that is, the "representation" of those organ functions in our brain matter; or, in other words, the neurological and chemical processing related to that organ) was utilized by our mind to process those new thoughts. And this produced the close association between our abdominal organs and our thoughts [1].

For example, in our body, our liver is responsible for organizing the supply of energy and for creatively adjusting that supply, as needed. And in our mental world it is our liver that is responsible for causing us to organize the world around us, and to produce creative solutions to problems. But when one of those organizational thoughts is blocked by our conscious mind, such as when we see someone breaking a rule (being unfair, treating us wrongly, or speaking to us inappropriately), or not doing something in the way we think it should be done, this "blockage" in our liver-related thoughts causes our physical liver's physical function to also be immediately blocked, which then causes our liver to produce that emotion of irritation or rage that we feel in any of these situations. If this situation continues for some time, our blocked liver function causes all the common symptoms of stress within us, such as a stiff neck and shoulders, constant irritability or rage (which we often attempt to suppress) or, in some people, even eventually a migraine [1].

\section{Our Organs Drove the Evolution of Our Body}

All this evidence suggests that it was our main abdominal organs that guided the evolution of our body, senses, and thoughts. If this was the case, we would expect all creatures that have the same organs, to also have a very similar bodily structure and senses. And this is exactly what exists; whereas, creatures with different organs, such as jelly fish, have a totally different bodily structure.

It is probable that the digestive organs began to evolve first, which produced the energy and other resources that were needed to then enable a complex body to form; and it is possible that the body then gradually took on the shape and design that exists today, in response to information from its own organs.

If this was the case, all this was made possible by two simple accidents. The first, that organ information is superimposed on the electrical current that seems to be an integral part of all living tissue, so that this organ information was conveyed to every part of a creature's body. And the second accident was that bodily tissue was able to interpret this information and produce changes in itself to reflect that infor- mation. This determined that over millions of years, our body would evolve in response to this information. In other words, a creature's body evolved to serve its own abdominal organs.

Evolution was not accidental, though the concept of natural selection may have played a helping hand to guide a creature in a particular direction. As to the genetic code, the author speculates that this is simply a reflection of each creature's current state, which then enables that state to be passed on to the next generation. But it was not a guiding force in itself.

Today's main approach to healthcare considers that our body is merely a machine that serves the brain. In other words, that the body is brain-centric. But the truth is that our body is very much more organ-centric. Our abdominal organs have a profound influence on our every thought and emotion, and also on the good functioning of our body. When one of our organs is stressed, this produces marked anomalies in our thoughts and emotions, and also pains and visible anomalies along the sections of the body that resonate with that organ (in other words, the organ's meridian).

\section{What are the Implications for Healthcare?}

To correct most health issues, it is only necessary to prompt the organs to correct their own malfunctions; and the ancient Chinese discovered a very effective way of doing this. Over thousands of years, Chinese physicians noticed that almost all symptoms, and also many unhealthy thought patterns, are directly produced by one or more of our abdominal organs being stressed. This stress is interpreted by our bodily tissue, which then produces the physical symptoms. Any of these health issues can be readily corrected by stimulating the points on the body that resonate with the stressed organs (i.e. points on their meridians).

For example, to help a patient recover from a respiratory condition, such as COVID-19, it is only necessary to stimulate lung and "kidney" acupoints, which then immediately strengthen the lung function and enable the patient to recover from the condition, just as they would from a common cold.

Hopefully, the world will soon realize that today's preoccupation with doing battle with pathogens is a medical red herring; and that it is not necessary to bring the whole world to its knees, socially and economically, simply because today's mainstream medical industry cannot understand the body's own powerful healing mechanisms.

\section{Conflict of Interest Statement}

The author received no financial contribution towards any aspect of the research described in this article, nor towards the preparation of this article.

\section{References}

1. Kovich F (2019) Acupuncture today and in ancient China. Curious Pages Publishing Bristol, United Kingdom.

2. Kovich F (2019) The lungs' real-time states are reflected in the tissue at its related acupuncture points. J Acupunct Res 36: 88-91.

3. Kovich $F$ (2019) The stomach's communication with its related acupoints, and the "intelligent tissue" hypothesis. J Acupunct Res 36: 21-27. 